\title{
FACTORS INFLUENCING CURRICULUM ADOPTION IN UNDERGRADUATE CYBERSECURITY PROGRAMS
}

\author{
Todd A. Whittaker, Franklin University, todd.whittaker@franklin.edu \\ Cherie Noteboom, Dakota State University, cherie.noteboom@dsu.edu
}

\begin{abstract}
Increased demand in the cybersecurity workforce requires a significant response from colleges and universities to meet that demand. The federal government has emphasized cybersecurity education at all levels as a way to address demand, yet there is wide variance in curriculum defined by academics, industry, and government organizations. While there are many curriculum standards, little research has been conducted to investigate the drivers for curriculum adoption. This study aims to discover what factors influence the adoption of new curriculum at the undergraduate level through a quantitative adaptation and application of existing technology adoption models (e.g. UTAUT, UTAUT2, TRA, TPB, TAM) to the domain of curriculum adoption. It is hypothesized that many of the same factors that drive technology adoption also drive curriculum adoption with the addition of altruistic motivation of the faculty member on behalf of the student. The survey-based study employs a path model analyzed using partial least squares structural equation modeling (PLS-SEM). If it is desirable to move toward standardized cybersecurity curriculum or to encourage faculty to adopt existing high quality curriculum, this work will benefit standards bodies, accreditors, university leaders, and the federal government to determine the factors that drive adoption to direct resources appropriately.
\end{abstract}

Keywords: Cybersecurity, Curriculum, Technology Acceptance Models, Technology Adoption

\section{INTRODUCTION AND MOTIVATION}

The US federal government is investing millions of dollars in building a strong cyber workforce capable of meeting the demands of industry and government for highly educated and effective security professionals. Initiatives such as the National Initiative for Cybersecurity Education (National Institute of Standards and Technology, 2016), the NICE Cybersecurity Workforce Framework (Newhouse, Keith, Scribner, \& Witte, 2016), and the joint DHS/NSA National Centers for Academic Excellence in Cybersecurity (National Security Agency, n.d.) are a few of the ways the federal government is attempting to meet that need.

Among the many challenges are the competing standards for curriculum in cybersecurity. The NSA guidelines for their Centers of Excellence describes high-level topics and outcomes. The Association for Computing Machinery (ACM) Joint Task Force on Cybersecurity Education has developed its own guide (Burley et al., 2017), as has the Accreditation Board for Engineering and Technology (ABET) (Phillips et al., 2016). Industry certifications such as (ISC) ${ }^{2}$ 's CISSP and CompTIA's Security+ body of knowledge documents represent another body of knowledge. While there is substantial overlap among the standards, it is cumbersome for faculty to juggle competing criteria or create complex mappings. As a result, curriculum varies widely.

Faculty must choose what kinds of topics to include and little has been written concerning their curricular decisionmaking process. Faculty are critical to keeping curriculum current as regional accrediting bodies require substantive faculty oversight of curriculum in a shared governance model - e.g. HLC (2018). At a time when many colleges are adding some cyber-related topics, understanding the factors that drive changes in existing curriculum will help government, academic, and industry standardization efforts to direct resources appropriately to colleges.

The research question investigated is: what factors influence the cybersecurity curricular decisions of faculty in undergraduate programs? By surveying faculty with responsibility for current cybersecurity curriculum and using an adaptation of the Unified Theory of Acceptance and Use of Technology version 2 (UTAUT2), the goal of this research is to identify and quantify the precursors of curriculum adoption behavior of faculty in undergraduate programs. 


\section{Issues in Information Systems}

Volume 20, Issue 3, pp. 64-73, 2019

\section{LITERATURE REVIEW}

There have been many calls for cybersecurity curriculum standards in the past decade and much has been published about the need for a common body of knowledge and what its elements should be. Most, if not all, standards focus on the bachelors or masters levels.

What is curriculum? Defining curriculum is a difficult task fraught with confusion. As noted by Egan (1978)

At a superficial level, confusion about what curriculum is, and thus what people concerned with it should do, involves argument about whether curriculum subsumes instruction... or whether curriculum involves all learning experiences, or refers simply to a blueprint for achieving restricted objectives in a school setting, or includes the statement of objectives as well, or also the evaluation of their achievement, and so on. The field seems to have no clear logical boundaries.

Thus, curriculum could be narrowly defined to outcomes or broadly defined to include all aspects of the program of study with which a student engages. However, for this research, curriculum will be defined as any designed set of educational experiences. Within the context of cybersecurity, this could range from a set of lab exercises, readings from textbooks, a course, certificate, minor, or up to an entire program of study for a degree.

Much of the existing literature on cybersecurity curriculum is devoted to understanding the body of knowledge that is cybersecurity. Notable examples are Theoharidou and Gritzalis (2007), Manson, Curl, and Torner (2009), Maconachy, Duryea, and Starland (2009), and Bishop and Taylor (2009). However, all these studies focus on what to teach or what is taught based on standards and frameworks - none describe why curriculum decisions are made.

\section{Behavioral and Technology Acceptance Models}

To identify factors influencing intention toward a behavior, it is appropriate to discuss the relevant information systems theories for technology acceptance and then adapt those to curriculum adoption. The four most relevant theories for this study are the Theory of Reasoned Action (TRA), the Theory of Planned Behavior (TPB), the Technology Acceptance Model (TAM), and the Unified Theory of Acceptance and Use of Technology (UTAUT and UTAUT2). Each is discussed below.

First proposed by Fishbein and Ajzen (1975), the Theory of Reasoned Action consists of four constructs: behavior, behavioral intention, attitude toward behavior, and the subjective norms about the behavior. As a causal model, attitudes and norms drive intention, and intention drives behavior. Under TRA, both attitude and subjective norms influence the intention of a person to carry out a specific action. However, intent is not a guarantee of the behavior occurring. External constraints (e.g. resources, time, training, etc.) will further impact the probability of carrying out the actual behavior. It was precisely this limitation that drove the development of the Theory of Planned Behavior.

Proposed by Ajzen (1985), the Theory of Planned Behavior attempts to address the external influences not accounted for in TRA by adding the additional construct of "Perceived Behavioral Control," as a precursor to behavioral intention and actual behavior. As such, "perceived behavioral control reflects motivational factors that have an indirect effect on behavior through intentions. . . [and] reflects actual control and has a direct link to behavior not mediated by intentions," (Madden, Ellen, \& Ajzen, 1992).

The Technology Acceptance Model was another improvement on TRA. Developed by Davis (1989), TAM and its subsequent revisions employ the constructs of perceived usefulness and perceived ease of use as antecedents to attitude and behavioral intention and finally actual system use. Contrasted against TRA, TAM removes the attitude constructs and focuses on system-specific attributes. Perceived ease of use is "the degree to which a person believes that using a particular system would be free of effort," (Davis, 1989). Likewise, perceived usefulness is "the degree to which a person believes that using a particular system would enhance his or her job performance," (Davis, 1989). However, ease of use is also a potential system feature and would influence perceived usefulness and intention to use.

The Unified Theory of Acceptance and Use of Technology (UTAUT) was an attempt to join many of the existing theories of technology acceptance into a single theory. It built on TRA, TPB, and TAM in addition to other information systems and social science models. Venkatesh et al. (2003) built the model by first empirically measuring each of the eight models on data from four organizations. Using partial least squares, they tested for convergent and discriminant 


\section{Issues in Information Systems}

Volume 20, Issue 3, pp. 64-73, 2019

validity and determined loading factors for each of the constructs. The best of the individual models, TAM2, was able to account for approximately $53 \%$ of variance in actual system use.

By grouping similar constructs from the eight separate models, Venkatesh et al. was able to narrow the number of constructs down to seven which were used in an exploratory analysis. However, only four of the seven exogenous variables and the sole endogenous variable ("Behavioral Intention") was were used to build the final model, the analysis having revealed that the additional three did not add explanatory power. The final exogenous constructs were "Performance Expectancy," "Effort Expectancy," "Social Influence," and "Facilitating Conditions." Using this model Venkatesh et al. was able to explain $70 \%$ of variance - far more than any other single model of technology acceptance.

In 2012, Venkatesh et al. extended the earlier UTAUT model to UTAUT2 to study consumer behaviors related to technology acceptance. In the second iteration, constructs of "Hedonic Motivation," "Price Value," and "Habit." The final UTAUT2 model, shown in Figure 2, shows the seven exogenous variables and the two endogenous variables.

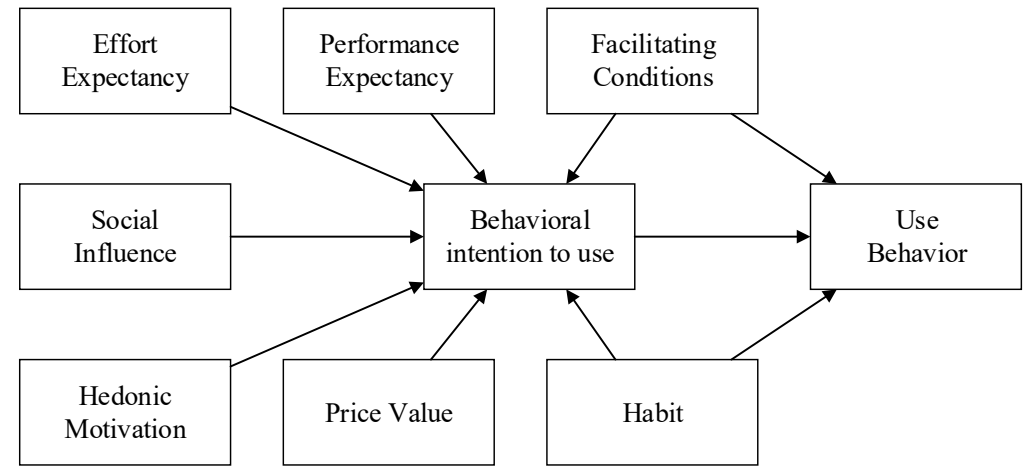

Figure 1. Unified Theory of Acceptance and Use of Technology (version 2) model

The additions of the three constructs "produced a substantial improvement in the variance explained in behavioral intention (56 percent to 74 percent) and technology use (40 percent to 52 percent)," (Venkatesh et al., 2012).

\section{Curriculum Adoption and Curriculum as Technology}

As mentioned previously, motivations for curriculum adoption has not been widely studied. However, one effort by $\mathrm{Ni}$ (2009) did consider factors influencing adoption of "curriculum innovation." Using other fields as a basis, Ni states that

No change can occur without the teacher believing that the change is worth making. From this perspective, teachers' knowledge and beliefs could serve as critical factors that impact teachers' decisions about whether to adopt a new curriculum, especially at the post-secondary level where teachers have significant influence (if not the final decision) over adoption.

$\mathrm{Ni}$ collected data about attitudes and beliefs of teachers regarding curriculum adoption in computer science. After careful analysis using a mixed-methods approach, Ni concluded that among the many factors influencing adoption the largest single predictor was teachers' excitement about the new approach, which "could predict $70.8 \%$ of actual adoption."

Curriculum has been studied as a technology (Jenkins, 2009; Johnson, 2015; Zuga, 1989). A behavioral model adapted from psychology, curriculum-as-technology forms a system in which the student-teacher feedback loop is employed to transmit knowledge and skills from teacher to student (Jenkins, 2009; Johnson, 2015). As an information system, curriculum consists of the people involved (students, teachers), the processes followed (instruction, assessment), the data that is processed (instructional content), and communications (student-teacher interactions). Therefore the adoption of curriculum is consistent with the adoption of an information system or technology and the models can be legitimately applied. 


\section{Issues in Information Systems \\ Volume 20, Issue 3, pp. 64-73, 2019}

\section{Student Performance Expectancy}

Performance expectancy has long been a part of most technology adoption models - whether captured as "attitude toward behavior," in TRA "perceived usefulness," in TAM or by the directly named "performance expectancy," construct in UTAUT andUTAUT2. However, all technology acceptance models and associated studies interpret performance expectancy from the perspective of the user of the system as "first person" performance expectancy. However, in teaching and learning situations (as is the case with curriculum), it isn't the instructor's expectation of his or her improved performance that is at issue. Instead, it is concern about the students' performance that is paramount.

Allen (2016 p. 88) makes this instructor focus on student performance clear - in this context, about e-learning: "It's easy to assume that e-learning is only about teaching things, but success isn't the result when people know the right things to do, yet continue to do the wrong things. [Learning] must be designed to enable, facilitate, and reward good performance." For Allen, typical learning experiences focus on content, information presentation, and knowledge acquisition whereas serious learning experiences target "performance outcomes," (2016 p. 112).

Although education, curriculum, and instruction literature posit instructor motive for student performance expectancy, the question remains as to whether the same case can be made in technology adoption models. No direct theory answers this question, but several others can be used to triangulate on one: Multimotive Information Systems Continuance Model (Lowry, Gaskin, \& Moody, 2015) and Stakeholder Theory (Freeman \& McVea, 2001).

Explored by Lowry et al, (2015), the multimotive information systems continuance model seeks to add to the existing theory of user acceptance (or in this case continuation of use) of information systems. Lowry et al. state that "most extant models of user perceptions and evaluations of information systems focus on fulfilling users' extrinsic motivations such as desires for productivity, efficiency, and general utility. These models, however, do not fully explain the range of intrinsic and extrinsic motivations that influence these outcome variables," (2015). The authors differentiate motivations for technology acceptance, including intrinsic, extrinsic, and hedonic motivations.

Extrinsic motivations are generally captured in existing models as first-party performance expectancy. Intrinsic motivations, however are internal rewards (e.g. personal satisfaction) for carrying out an action for its own sake and are more concerned with the process that leads to the outcome than the outcome itself (Lowry et al., 2015). Examples of intrinsic motivation are "accomplishment, learning or enlightenment, and socialization," (Lowry et al., 2015).

Lowry et al. constructed a taxonomy of intrinsic motivations. Those relevant to faculty motives for student performance are influencing others, altruism (including helping others learn), improving reputation or receiving approval, leadership, and knowledge sharing. Finally Lowry et al. specifically identifies the UTAUT model as an example "of how differentiation between types of intrinsic motivation can influence future research... on system adoption," (2015).

Stakeholder theory was first published by R.E. Freeman in 1984 as an alternative to the input/output theory of the firm and states that strategic decisions should address the key interests of all stakeholders of that firm. (Freeman \& McVea, 2001). Those interests are bi-directional - "each of these groups can be seen as supplying the firm with critical resources (contributions) and in exchange each expects its interests to be satisfied," (Hill \& Jones, 1992).

While colleges and universities are not "firms" in the same sense as Freeman \& McVea, they still do fit well within the framework of stakeholder theory - "meeting the needs of individuals or groups is an important competitive factor" for higher education institutions (Alves, Mainardes, \& Raposo, 2010). In all previous stakeholder studies in higher education, Alves et al. note, students, faculty, staff, alumni, government, and employers are all significant stakeholders whose interests must be considered.

When studying the motivations of students majoring in accounting, Byrne \& Flood (2005) found that "career and educational aspirations are the main reasons why these students choose to go to university." Four of the five highest scoring indicators in their research were related to opportunities, job performance, earning power, and useful career skills (Byrne \& Flood, 2005). Generalizing from accounting students, this demonstrates that many, if not most, students are attending universities for future performance expectancy. Under stakeholder theory, this is sufficient reason for faculty, as agents of the institution, to consider student performance expectancy as part of their curriculum adoption intent. 


\section{Issues in Information Systems}

Volume 20, Issue 3, pp. 64-73, 2019

\section{RESEARCH METHODOLOGY}

The unit of analysis in this research was an undergraduate faculty member or academic administrator with responsibility over a cybersecurity-related major, minor, certificate, or course. The survey-based instrument was based on UTAUT2 with the addition of student performance expectancy and was distributed through numerous cybersecurity mailing lists for undergraduate college faculty (e.g. 3CS, The National CyberWatch Center, CSSIA, CCW, BATEC). The operationalized constructs are given in Table 1 and arranged as a path model with hypotheses in Figure 2. Two additional hypotheses, $\mathrm{H} 10$ and $\mathrm{H} 11$ refer to the mediating effects of $\mathrm{FC}$ on $\mathrm{AB}$ via BIA and the effects of SPE on BIA via FPE respectively.

Table 1. Operationalized constructs in the model

\begin{tabular}{|c|c|c|}
\hline Construct & Definition & Theory \\
\hline $\begin{array}{l}\text { Student } \\
\text { Performance } \\
\text { Expectancy (SPE) }\end{array}$ & $\begin{array}{l}\text { The degree to which an individual believes that } \\
\text { adopting the curriculum will help his or her students } \\
\text { to attain gains in job performance. }\end{array}$ & $\begin{array}{l}\text { Allen (2016), Lowry et al. (2015), } \\
\text { Freeman and McVea (2001), } \\
\text { Byrne and Flood (2005) }\end{array}$ \\
\hline $\begin{array}{l}\text { Faculty Performance } \\
\text { Expectancy (FPE) }\end{array}$ & $\begin{array}{l}\text { The degree to which an individual believes that } \\
\text { adopting the curriculum will help him or her to attain } \\
\text { gains in job performance. }\end{array}$ & Venkatesh et al. (2003) \\
\hline $\begin{array}{l}\text { Effort Expectancy } \\
\text { (EE) }\end{array}$ & $\begin{array}{l}\text { The degree of ease associated with the adoption of the } \\
\text { curriculum }\end{array}$ & Venkatesh et al. (2003) \\
\hline Social Influence (SI) & $\begin{array}{l}\text { The degree to which an individual perceives that } \\
\text { important others believe he or she should adopt the } \\
\text { curriculum. }\end{array}$ & Venkatesh et al. (2003) \\
\hline $\begin{array}{l}\text { Facilitating } \\
\text { Conditions (FC) }\end{array}$ & $\begin{array}{l}\text { The degree to which an individual believes that an } \\
\text { organizational and technical infrastructure exists to } \\
\text { support the adoption of the curriculum. }\end{array}$ & Venkatesh et al. (2003) \\
\hline $\begin{array}{l}\text { Hedonic Motivation } \\
\text { (HM) }\end{array}$ & $\begin{array}{l}\text { The degree to which an individual believes that the } \\
\text { adoption of the curriculum will be enjoyable. }\end{array}$ & $\begin{array}{l}\text { Venkatesh et al. (Venkatesh et al., } \\
\text { 2012), Ni (2009) }\end{array}$ \\
\hline $\begin{array}{l}\text { Behavioral Intention } \\
\text { to Adopt (BIA) }\end{array}$ & $\begin{array}{l}\text { The degree to which an individual intends to adopt } \\
\text { the new curriculum. }\end{array}$ & Venkatesh et al. (2003) \\
\hline $\begin{array}{l}\text { Adoption Behavior } \\
\text { (AB) }\end{array}$ & $\begin{array}{l}\text { The degree to which an individual adopts the } \\
\text { curriculum according to intention. }\end{array}$ & Venkatesh et al. (2003) \\
\hline
\end{tabular}

Indicator questions were presented in a 7-point Likert scale from "strongly agree," to "strongly disagree" and are based on published sources from Venkatesh et al. (2003) and Venkatesh et al. (2012). Questions were contextualized from technology acceptance to curriculum adoption - e.g. performance expectancy was changed from "I would find the system useful in my job," to "My students would find the curriculum useful in their future jobs," for student performance expectancy and to "I would find the curriculum useful in my job," for faculty performance expectancy. Demographic moderator information from participants consistent with Venkatesh et al (2003) were also gathered.

\section{RESULTS AND DISCUSSION}

More than 100 survey responses were received, but only 57 were complete and used for analysis via partial least squares structural equation modeling (PLS-SEM) software - SmartPLS. Following the process in Hair et al. (2017), this section evaluates the reflective measurement model for reliability, convergent validity, and discriminant validity. This is followed by an evaluation of the structural, or path model, to assesses the relationships between the constructs to calculate the coefficients of determination $\left(R^{2}\right)$, predictive relevance $\left(Q^{2}\right)$, and effect sizes $\left(f^{2}\right.$ and $\left.q 2\right)$.

\section{Measurement Model Evaluation}

The initial loadings of the indicators on the latent variables is calculated through the PLS algorithm. Five of the indicators were significantly cross-loaded on other constructs and were eliminated. One indicator (SI_3) was determined to measure the FC construct. While the indicator in question involves social influence, it fit better as a facilitating condition and thus the theory supports moving this indicator. When the analysis was rerun with the SI_3 
indicator on FC, the loading on FC increased substantially with relatively minor crossloadings on other constructs. Table 2 captures the final loadings and crossloadings of the measurement model.

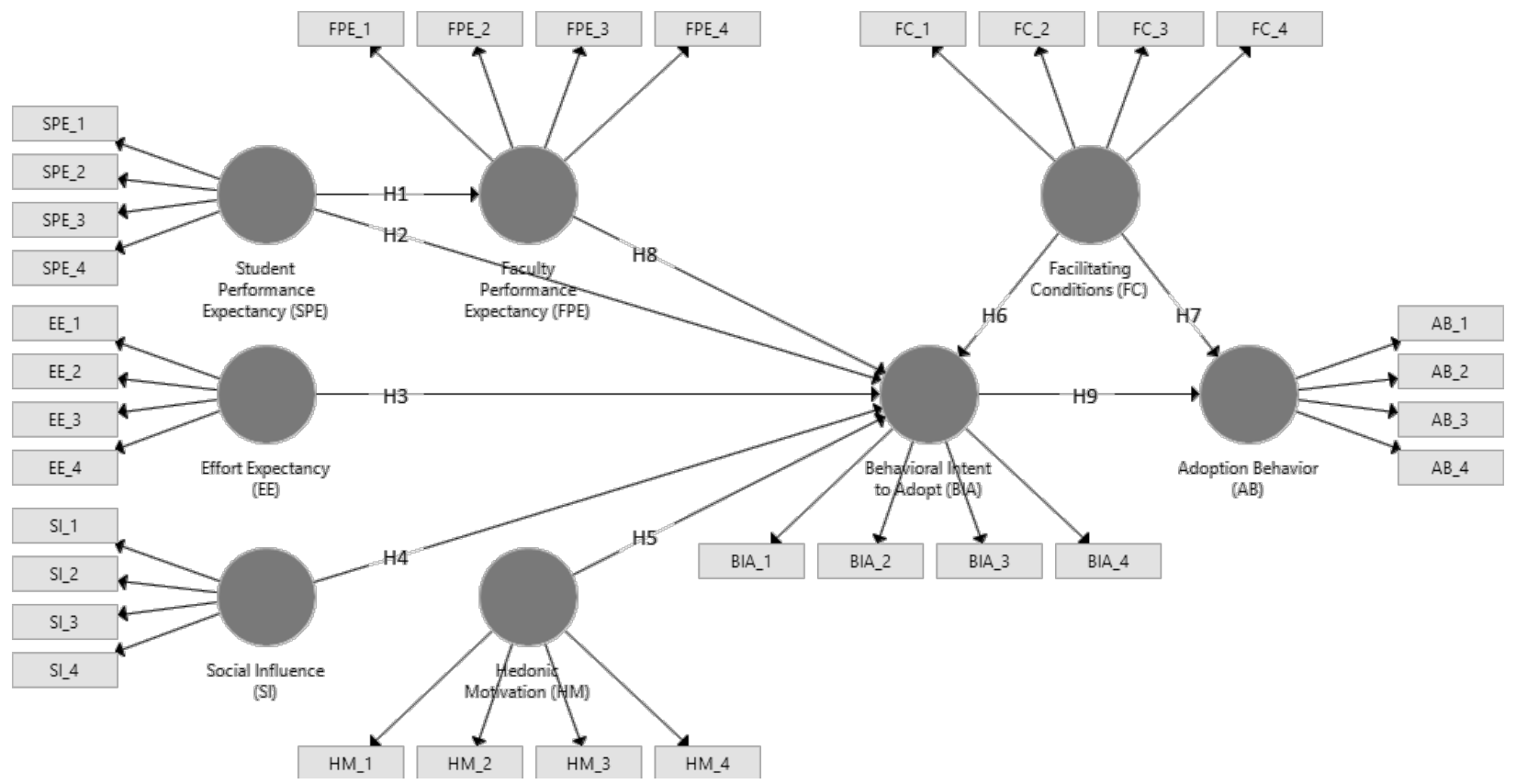

Figure 2. Measurement and path model

Construct reliability, validity, and internal consistency is established through Cronbach's alpha, composite reliability, and average variance extracted (AVE); see Table 3. All constructs had significant $(p<0.05)$ AVE based on indicators. Only the EE construct has a low Cronbach's alpha, but composite reliability and AVE are all within acceptable ranges.

Discriminant validity has traditionally been established by checking crossloadings of indicators on other constructs. Note that in Table 2 each indicator loads highly (more than 0.708 ) on its associated variable and has no crossloadings within 0.2 of another variable. The current practice in PLS-SEM favors the heterotrait-monotrait ratio (HTMT) over the Fornell-Larker criterion. HTMT is "the ratio of the between-trait correlations to the within-trait correlations," (Hair et al., 2017 p. 118). A threshold value of 0.9 is typically used; any ratio above 0.9 indicates lack of discriminant validity between constructs. Table 4 shows that the constructs are distinct from one another.

\section{Structural Model Evaluation}

In covariance-based structural equation modeling, there would be a step to assess "goodness-of-fit" of the model which contrasts the covariance matrix of the model against the covariance matrix of the samples and yielding a $\chi^{2}$ statistic. However, partial least squares is based on maximizing prediction via goal-seeking to "maximize the explained variance instead of minimizing the differences between covariance matrices," (Hair et al., 2017 p. 192). Thus, path coefficients, $R^{2}$ values, $f^{2}$ effect size, $Q^{2}$ predictive relevance, and $q^{2}$ effect size are considered the best way of evaluating the structural model.

The structural model is evaluated via the path coefficients and significance ( $p$-values) for each of those paths. Coefficients are determined by the PLS algorithm while significance is determined by samples via the bootstrapping procedure. The effect size, $t$-statistics, $p$-values, and confidence intervals are given in Table 5 . Note that if there is a direct path between constructs in Figure 2, the "effect size" is the path coefficient, otherwise it is the total effect through all paths.

All effects from SPE are significant as is the direct effect of BIA on $\mathrm{AB}$ and $\mathrm{FC}$ on $\mathrm{AB}$. Paths from SI to BIA and $\mathrm{AB}$ bear further investigation as does the path from FC to BIA. It could be that these become significant in a more parsimonious model. 
Table 2. Final loadings of indicators. Highest loading construct is in bold.

\begin{tabular}{lcccccccc}
\hline & AB & BIA & EE & FC & FPE & HM & \multicolumn{1}{c}{ SI } & \multicolumn{1}{l}{ SPE } \\
\hline AB3 & $\mathbf{0 . 9 2 3}$ & 0.613 & 0.231 & 0.515 & 0.371 & 0.268 & 0.452 & 0.311 \\
AB4 & $\mathbf{0 . 9 0 2}$ & 0.595 & 0.176 & 0.396 & 0.571 & 0.308 & 0.389 & 0.451 \\
BIA1 & 0.571 & $\mathbf{0 . 8 4 9}$ & 0.255 & 0.282 & 0.522 & 0.328 & 0.449 & 0.426 \\
BIA3 & 0.619 & $\mathbf{0 . 8 7 5}$ & 0.145 & 0.269 & 0.366 & 0.105 & 0.349 & 0.410 \\
BIA4 & 0.536 & $\mathbf{0 . 8 8 6}$ & 0.143 & 0.305 & 0.359 & 0.267 & 0.295 & 0.446 \\
EE1 & 0.124 & 0.157 & $\mathbf{0 . 7 6 7}$ & 0.332 & 0.038 & 0.133 & -0.032 & -0.044 \\
EE3 & 0.229 & 0.183 & $\mathbf{0 . 8 3 3}$ & 0.343 & 0.408 & 0.354 & 0.024 & 0.217 \\
FC1 & 0.194 & 0.041 & 0.431 & $\mathbf{0 . 7 4 2}$ & 0.111 & 0.430 & -0.061 & 0.025 \\
FC4 & 0.505 & 0.345 & 0.267 & $\mathbf{0 . 9 1 3}$ & 0.172 & 0.365 & 0.285 & 0.132 \\
SI3 & 0.473 & 0.320 & 0.478 & $\mathbf{0 . 9 2 4}$ & 0.252 & 0.498 & 0.313 & 0.155 \\
FPE1 & 0.582 & 0.487 & 0.307 & 0.374 & $\mathbf{0 . 9 1 4}$ & 0.445 & 0.301 & 0.612 \\
FPE2 & 0.444 & 0.441 & 0.127 & 0.040 & $\mathbf{0 . 8 8 3}$ & 0.480 & 0.212 & 0.678 \\
FPE3 & 0.339 & 0.374 & 0.371 & 0.185 & $\mathbf{0 . 8 9 4}$ & 0.571 & 0.171 & 0.632 \\
HM1 & 0.086 & 0.048 & 0.182 & 0.344 & 0.357 & $\mathbf{0 . 8 5 4}$ & 0.013 & 0.351 \\
HM2 & 0.129 & 0.145 & 0.363 & 0.479 & 0.452 & $\mathbf{0 . 8 7 4}$ & 0.041 & 0.411 \\
HM3 & 0.210 & 0.174 & 0.263 & 0.366 & 0.344 & $\mathbf{0 . 8 7 1}$ & -0.053 & 0.371 \\
HM4 & 0.411 & 0.353 & 0.274 & 0.460 & 0.617 & $\mathbf{0 . 9 3 7}$ & 0.170 & 0.621 \\
SI1 & 0.438 & 0.338 & -0.019 & 0.260 & 0.259 & 0.080 & $\mathbf{0 . 9 6 4}$ & 0.211 \\
SI2 & 0.460 & 0.468 & 0.010 & 0.281 & 0.241 & 0.091 & $\mathbf{0 . 9 8 2}$ & 0.267 \\
SPE1 & 0.419 & 0.558 & 0.073 & 0.121 & 0.675 & 0.501 & 0.229 & $\mathbf{0 . 9 3 0}$ \\
SPE2 & 0.317 & 0.360 & 0.065 & 0.061 & 0.696 & 0.528 & 0.187 & $\mathbf{0 . 9 4 2}$ \\
SPE3 & 0.492 & 0.514 & 0.120 & 0.171 & 0.594 & 0.463 & 0.310 & $\mathbf{0 . 9 1 3}$ \\
SPE4 & 0.306 & 0.386 & 0.189 & 0.177 & 0.692 & 0.570 & 0.204 & $\mathbf{0 . 9 3 5}$ \\
\hline
\end{tabular}

Table 3. Construct reliability and validity.

\begin{tabular}{lccc}
\hline & Cronbach & Composite & AVE \\
\hline AB & 0.800 & 0.909 & 0.833 \\
BIA & 0.840 & 0.904 & 0.758 \\
EE & 0.442 & 0.781 & 0.641 \\
FC & 0.845 & 0.897 & 0.746 \\
FPE & 0.879 & 0.925 & 0.805 \\
HM & 0.924 & 0.934 & 0.780 \\
SI & 0.945 & 0.973 & 0.947 \\
SPE & 0.948 & 0.962 & 0.865 \\
\hline
\end{tabular}

Table 4. HTMT discriminant validity; all values $<0.9$.

\begin{tabular}{lccccccc}
\hline & AB & BIA & EE & FC & FPE & HM & SI \\
\hline AB & - & - & - & - & - & - & - \\
BIA & 0.806 & - & - & - & - & - & - \\
EE & 0.367 & 0.341 & - & - & - & - & - \\
FC & 0.539 & 0.325 & 0.737 & - & - & - & - \\
FPE & 0.612 & 0.554 & 0.519 & 0.250 & - & - & - \\
HM & 0.269 & 0.244 & 0.455 & 0.544 & 0.546 & - & - \\
SI & 0.528 & 0.458 & 0.053 & 0.280 & 0.280 & 0.082 & - \\
SPE & 0.479 & 0.547 & 0.258 & 0.144 & 0.782 & 0.520 & 0.260 \\
\hline
\end{tabular}

The coefficient of determination $\left(R^{2}\right)$ measures the predictive power of a model for the endogenous variables and roughly estimates the variance explained by the model for that endogenous construct; see Table 6 . Using the categories recommended by Urbach and Ahlemann (2010), values around 0.670 are considered substantial, 0.333 are moderate, and 0.190 and lower are weak. In both the $R^{2}$ values of AB and FPE are in the substantial range while BIA is in the moderate range. Roughly $51 \%$ of adoption behavior can be explained by the model of Figure 2 and it is concluded that this model has between moderate and substantial predictive power

The predictive relevance, or $Q^{2}$ value, is "an indicator of the model's out-of-sample predictive power or predictive relevance," (J. F. Hair et al., 2017). For each endogenous variable, a $Q^{2}$ value greater than zero indicates predictive relevance. $Q^{2}$ is calculated by a "blindfolding" procedure by systematically eliminating every $n^{\text {th }}$ indicator data point (where $n$ is the "omission distance"). If the average replacement predicts the missing values, a non-zero $Q^{2}$ is the result; See Table 6 . Since the $Q^{2}$ values are significantly different from zero, the model has high predictive relevance. 
Table 5. Total effects and significance.

\begin{tabular}{lrrrrcc}
\hline & Effect size & $t$-statistic & $p$-value & $\begin{array}{c}95 \% \text { Confidence } \\
\text { Intervals }\end{array}$ & Hypothesis & $\begin{array}{c}\text { Significant } \\
(p<0.05) ?\end{array}$ \\
\hline $\mathrm{BIA} \rightarrow \mathrm{AB}$ & 0.559 & 6.036 & 0.000 & {$[0.346,0.723]$} & $\mathrm{H} 9$ & Yes \\
$\mathrm{EE} \rightarrow \mathrm{AB}$ & 0.042 & 0.473 & 0.636 & {$[-0.129,0.216]$} & - & No \\
$\mathrm{EE} \rightarrow \mathrm{BIA}$ & 0.075 & 0.491 & 0.623 & {$[-0.232,0.357]$} & $\mathrm{H} 3$ & No \\
$\mathrm{FC} \rightarrow \mathrm{AB}$ & 0.446 & 4.105 & 0.000 & {$[0.249,0.678]$} & $\mathrm{H} 7$ & Yes \\
$\mathrm{FC} \rightarrow \mathrm{BIA}$ & 0.228 & 1.653 & 0.098 & {$[-0.017,0.518]$} & H6 & No \\
$\mathrm{FPE} \rightarrow \mathrm{AB}$ & 0.114 & 0.922 & 0.357 & {$[-0.191,0.305]$} & - & No \\
$\mathrm{FPE} \rightarrow \mathrm{BIA}$ & 0.204 & 0.951 & 0.342 & {$[-0.306,0.534]$} & H8 & No \\
$\mathrm{HM} \rightarrow \mathrm{AB}$ & -0.108 & 1.020 & 0.308 & {$[-0.318,0.091]$} & - & No \\
$\mathrm{HM} \rightarrow \mathrm{BIA}$ & -0.194 & 1.065 & 0.287 & {$[-0.549,0.159]$} & H5 & No \\
$\mathrm{SI} \rightarrow \mathrm{AB}$ & 0.132 & 1.339 & 0.181 & {$[-0.031,0.351]$} & - & No \\
$\mathrm{SI} \rightarrow \mathrm{BIA}$ & 0.236 & 1.582 & 0.114 & {$[-0.062,0.523]$} & H4 & No \\
$\mathrm{SPE} \rightarrow \mathrm{AB}$ & 0.279 & 2.075 & 0.038 & {$[0.015,0.519]$} & - & Yes \\
$\mathrm{SPE} \rightarrow \mathrm{BIA}$ & 0.498 & 2.069 & 0.039 & {$[0.022,0.894]$} & H2 & Yes \\
$\mathrm{SPE} \rightarrow \mathrm{FPE}$ & 0.715 & 4.347 & 0.000 & {$[0.250,0.914]$} & H1 & Yes \\
\hline
\end{tabular}

The effect size $\left(f^{2}\right)$ is an estimation of the contribution of an exogenous construct on its associated endogenous constructs and is calculated by comparing the endogenous variable's $R^{2}$ when the exogenous construct is both included and excluded from the model. Values of $0.02,0.15$, and 0.35 represent small, medium and large effects respectively (J. F. Hair et al., 2017). Table 7 shows the effect sizes for the model of Figure 2. BIA has a very large effect on AB, FC has a medium effect on AB, and SPE has an extreme effect on FPE. The constructs FC, FPE, HM, SI, and SPE all have small effects on BIA, and EE has no effect on BIA

Table 6. $R^{2}$ and $Q^{2}$ values

\begin{tabular}{lccc}
\hline & $R^{2}$ & $R^{2}$ adj. & $Q^{2}$ \\
\hline AB & 0.531 & 0.512 & 0.399 \\
BIA & 0.408 & 0.334 & 0.249 \\
FPE & 0.511 & 0.502 & 0.379 \\
\hline
\end{tabular}

Table 7. $R^{2}$ effect sizes $\left(f^{2}\right)$.

\begin{tabular}{lccc}
\hline & AB & BIA & FPE \\
\hline BIA & 0.594 & - & - \\
EE & - & 0.007 & - \\
FC & 0.193 & 0.051 & - \\
FPE & - & 0.029 & - \\
HM & - & 0.030 & - \\
SI & - & 0.075 & - \\
SPE & - & 0.088 & 1.044 \\
\hline
\end{tabular}

Table 8. $Q^{2}$ effect sizes $\left(q^{2}\right)$.

\begin{tabular}{lccc}
\hline & AB & BIA & FPE \\
\hline BIA & 0.077 & - & - \\
EE & - & -0.001 & - \\
FC & 0.090 & 0.029 & - \\
FPE & - & 0.003 & - \\
HM & - & 0.012 & - \\
SI & - & 0.035 & - \\
SPE & - & 0.045 & 0.264 \\
\hline
\end{tabular}

Just as $f^{2}$ measures the $R^{2}$ effect size, so does $q^{2}$ measure the $Q^{2}$ effect size. The calculation for $q^{2}$ is analogous to that of $f^{2}$ as is the interpretation of effect size; see Table 8. BIA and $\mathrm{FC}$ have a medium-small predictive relevance effect on AB. Further, FC, SI, and SPE have a small predictive relevance effect on BIA. Finally, SPE has a medium large predictive relevance effect on FPE. However, EE, FPE, and HM have no significant predictive relevance on BIA.

\section{Hypothesis Results and Discussion}

Based on the data in Table 5 and Table 8, the hypotheses can be evaluated. If the $p$-value of the effect size was significant and there was a direct path in Figure 2, then the hypotheses were supported. The supported hypotheses were $\mathrm{H} 1, \mathrm{H} 2, \mathrm{H} 7$, and $\mathrm{H} 9$. Hypotheses H3, H4, H5, H6, H8, H10, and $\mathrm{H} 11$ were not supported.

From the results above some conclusions can be drawn about what motivates faculty to adopt curriculum. First, the largest effect size of an exogenous construct is that of facilitating conditions - whether the organization provided the support needed to make a curriculum change. The second largest effect size is that of student performance expectancy - the faculty member's estimate of the future performance of students based on the change being made. It was surprising that social influence did not play a greater role; in a parsimonious model, SI could become significant.

Equally satisfying were those paths that were not significant: it didn't matter to faculty that the change was a lot of work (effort expectancy) or if they had fun doing it (hedonic motivation) or if there were extrinsic rewards (faculty 


\section{Issues in Information Systems}

Volume 20, Issue 3, pp. 64-73, 2019

performance expectancy). And although extrinsic rewards weren't significant in predicting adoption behavior, faculty did see that their success was tied to student success through the SPE $\rightarrow$ FPE path which had the largest effect size and predictive relevance between exogenous and endogenous constructs.

\section{CONCLUSION AND FUTURE WORK}

This research contains three significant contributions to the field of information systems and cybersecurity education. The first is the result itself - faculty are motivated by student performance expectancy and facilitating conditions. Efforts by standards bodies, accreditors, university leaders, and the federal government to drive curriculum change should focus on making the case about improved student outcomes and funding to drive change. The second contribution is that this research represents a first step toward a curriculum adoption (or acceptance) model that parallels that of technology acceptance. The "curriculum as technology" approach is valid in that the model of Figure 2 can account for $51 \%$ of actual adoption behavior through the given constructs. The third is that the model contains a unique and significant construct for third-party performance expectancy in student performance expectancy. Future work on this model will explore the "curriculum as technology" contribution by eliminating insignificant paths and constructs to produce a more parsimonious model with equal explanatory power. Moderating effects of experience level, age, associate's vs. bachelor's level institutions, highest degree earned, and the voluntariness of the change will also be considered. Other future contributions will explore the third party performance expectancy as an intrinsic motivator. Other intrinsic motivations such as learning and influence could also be explored.

\section{REFERENCES}

Ajzen, I. (1985). From Intentions to Actions: A Theory of Planned Behavior. In Action Control (pp. 11-39). Springer.

Allen, M. W. (2016). Michael Allen's Guide to e-Learning: Building Interactive, Fun, and Effective Learning Programs for Any Company, 2nd Edition. John Wiley \& Sons.

Alves, H., Mainardes, E. W., \& Raposo, M. (2010). A Relationship Approach to Higher Education Institution Stakeholder Management. Tertiary Education and Management, 16(3), 159-181.

Bishop, M., \& Taylor, C. (2009). A Critical Analysis of the Centers of Academic Excellence Program. Proceedings of the 13th Colloquium for Information Systems Security Education, 24-31.

Burley, D. L., Bishop, M., Buck, S., Ekstrom, J. J., Futcher, L., Gibson, D., ... Parrish, A. (2017). Cybersecurity Curricula 2017 (Vol. 1). Retrieved from 4594

Byrne, M., \& Flood, B. (2005). A study of accounting students' motives, expectations and preparedness for higher education. Journal of Further and Higher Education, 29(2), 111-124.

Davis, F. D. (1989). Perceived Usefulness, Perceived Ease Of Use, And User Acceptance. MIS Quarterly, 13(3), 319-339. Retrieved from http://www.jstor.org/stable/10.2307/249008

Egan, K. (1978). What Is Curriculum? Curriculum Inquiry, 8(1), 9-16.

Fishbein, M., \& Ajzen, I. (1975). Belief, Attitude, Intention, and Behavior, An Introduction to Theory and Research. Belief, Attitude, Intention, and Behavior, An Introduction to Theory and Research. Retrieved from http://people.umass.edu/aizen/f\&a1975.html

Freeman, R. E., \& McVea, J. F. (2001). A Stakeholder Approach to Strategic Management. University of Virginia, Darden Graduate School of Business Administration Working Paper 01-02.

Hair, J. F., Hult, G., Tomas, M., Ringle, C., \& Sarstedt, M. (2017). A primer on partial least squares structural equation modeling (PLS-SEM). Sage.

Hill, C. W. L., \& Jones, T. M. (1992). Stakeholder-agency theory. Journal of Management Studies, 29(2), 121-154.

HLC. (2018). Criteria for Accreditation -- Higher Learning Commission. Retrieved September 6, 2018, from 
https://www.hlcommission.org/Policies/criteria-and-core-components.html

Jenkins, S. B. (2009). Measuring teacher beliefs about curriculum orientations using the modified-curriculum orientations inventory. Curriculum Journal, 20(2), 103-120. Retrieved from http://www.tandfonline.com/doi/abs/10.1080/09585170902948798

Johnson, M. (2015). Orientations to Curriculum in Computer Art Education. Art Education, 50(3), 43-47.

Lowry, P. B., Gaskin, J. E., \& Moody, G. D. (2015). Proposing the Multimotive Information Systems Continuance Model (MISC) to Better Explain End-User System Evaluations and Continuance Intentions. Journal of the Association for Information Systems, 16(7), 515-579.

Maconachy, W. V, Duryea, J., \& Starland, P. (2009). An Analysis of the State of Cyber Security Education in America. Proceedings of the 13th Colloquium for Information Systems Security Education.

Madden, T. J., Ellen, P. S., \& Ajzen, I. (1992). A Comparison of the Theory of Planned Behavior and the Theory of Reasoned Action. Personality and Social Psychology Bulletin, 18(1), 3-9.

Manson, D. P., Curl, S. S., \& Torner, J. (2009). A Framework for Improving Information Assurance Education. Communications of the IIMA, 9(1), 79-90.

National Institute of Standards and Technology. (2016). The National Initiative for Cybersecurity Education (NICE). Retrieved July 11, 2016, from NIST Computer Security Resource Center website: http://csrc.nist.gov/nice/index.htm

National Security Agency. (n.d.). NIETP. Retrieved July 11, 2016, from The Information Assurance Directorate at the NSA website: https://www.iad.gov/NIETP/

Newhouse, B., Keith, S., Scribner, B., \& Witte, G. (2016). SP 800-181: National Initiative for Cybersecurity Education (NICE) Cybersecurity Workforce Framework (NCWF). NIST Special Publication, 130. Retrieved from http://csrc.nist.gov/publications/drafts/800-181/sp800_181_draft.pdf

Ni, L. (2009). What makes CS teachers change? Factors Influencing CS Teachers' Adoption of Curriculum Innovations. ACM SIGCSE Bulletin, 544-548. Retrieved from http://dl.acm.org/citation.cfm?id=1509051

Phillips, A., Blair, J., Parrish, A., Inglis, C., Fitsgerals, S., Burley, D., ... Lingafelt, C. S. (2016). An Interactive Workshop on Draft Program Criteria for Cyber Sciences. Retrieved from Cyber Education Project website: http://cep-public.caps.ua.edu/wp-content/uploads/2016/04/ABET-Symposium-CEP-materials-v4.pdf

Theoharidou, M., \& Gritzalis, D. (2007). Common body of knowledge for information security. Security \& Privacy, IEEE , 5, 64-67. Retrieved from http://ieeexplore.ieee.org/xpls/abs_all.jsp?arnumber=4140992

Urbach, N., \& Ahlemann, F. (2010). Structural Equation Modeling in Information Systems Research Using Partial Least Squares. Journal of Information Technology Theory and Application, 11(2), 5-40.

Venkatesh, V., Morris, M. G. ., Davis, G. B. ., \& Davis, F. D. . (2003). User Acceptance of Information Technology: Toward a Unified view. MIS Quarterly, 27(3), 425-478.

Venkatesh, V., Thong, J. Y. L., \& Xu, X. (2012). Consumer Acceptance and Use of Information Technology: Extending the Unified Theory of Acceptance and Use of Technology. MIS Quarterly, 36(1), 157-178.

Zuga, K. F. (1989). Relating technology education goals to curriculum planning. Journal of Technology Education, $1(1), 34-58$. 